\title{
La tolérance des Lumières à l'épreuve de la Révolution française
}

\author{
Paul CHOPELIN \\ Université Jean Moulin Lyon 3, LARHRA
}

La Révolution française est l'une des quatre grandes périodes d'affrontements religieux dans l'histoire de France, avec la crise religieuse du $\mathrm{II}^{\mathrm{e}}$ au $\mathrm{IV}^{\mathrm{e}}$ siècle dans la Gaule romaine (persécution des chrétiens, puis celle des païens), les guerres de religion au $\mathrm{XVI}^{\mathrm{e}}$ siècle et le duel Église/République des années 1880-1900. Le conflit religieux révolutionnaire est un phénomène complexe, souvent analysé à travers une grille de lecture héritée de la fin du XIX ${ }^{\mathrm{e}}$ siècle. Dans le cadre d'une interprétation progressiste de l'histoire, la Révolution est présentée comme un moment fondateur, qui réalise l'idéal de tolérance des Lumières en instaurant la liberté des cultes, dans le cadre de la Déclaration des Droits de l'Homme. À l'inverse, une historiographie conservatrice, de tradition catholique, insiste sur les persécutions de 1792-1794, pour mieux dénoncer l'hybris des révolutionnaires, qui voulaient établir une société nouvelle sur les ruines de l'Église. Ces deux traditions historiques s'opposent notamment sur la question de l'éventuelle intolérance religieuse des autorités républicaines entre 1792 et 1800 . Pour les défenseurs de la "Grande Révolution », la lutte contre le clergé réfractaire et ses fidèles aurait été motivée par leur collusion avec les forces royalistes, notamment lors des guerres de Vendée. Afin de rétablir l'ordre, la République n'aurait eu d'autre choix que de vouer ces perturbateurs du repos public à la mort ou à la déportation. Les historiens catholiques ont de leur côté dénoncé une persécution latente depuis les origines de la Révolution, fruit d'une culture anticléricale héritée des Lumières, portée par les chefs de file du mouvement révolutionnaire - principalement les Montagnards -, qui voulaient imposer par la force une sorte de laïcité républicaine avant la lettre. Ce cadre interprétatif fait d'ailleurs la part belle aux théories conspirationnistes quant aux origines « secrètes » de la Révolution.

Significativement, ces deux visions antagonistes de l'histoire religieuse de la Révolution se sont constituées au moment du conflit entre l'Église et la République au cours des années 1880-1900. Cet affrontement s'est aussi déroulé sur le terrain de l'histoire, à grands renforts de publications et de commémorations concurrentes. Depuis les lois contre les congrégations de 1880, les catholiques sont sur la défensive et sont persuadés de faire face à une nouvelle persécution, provoquée par les mêmes ennemis supposés qu'en 1789: les protestants et les francs-maçons. Au moment où la $\mathrm{III}^{\mathrm{e}}$ République s'apprête à fêter le centenaire de la "Grande Révolution », l'épiscopat décide de reconnaître officiellement le caractère de «martyrs » aux catholiques exécutés pour «fanatisme» ou refus de serment pendant la Révolution. Il s'agit, à terme, de mettre en place un cycle commémoratif susceptible de faire pièce aux célébrations officielles et de développer, chez les fidèles, une culture politique de résistance, appuyée sur l'histoire. Dès 1889, des contre-commémorations sont organisées un peu partout en France à l'initiative du clergé et des réseaux militants, pour exalter les figures de la France chrétienne « éternelle » que l'on oppose aux héros républicains promus par le régime. Parallèlement, la mémoire de la «persécution » est ranimée par de nouvelles recherches sur les «pieuses victimes » de la Terreur, donnant lieu à de multiples publications, généralement nourries d'une solide érudition, mais fortement orientées en défaveur de la Révolution. À travers l'histoire, les auteurs cherchent à mettre en garde les fidèles quant à une possible répétition de l'histoire : face aux lois «anticléricales », la mobilisation politique des catholiques est nécessaire pour éviter le déclenchement d'une nouvelle Terreur. Ces travaux fournissent les preuves nécessaires à la béatification des 
« martyrs de la Révolution », à commencer par les seize carmélites de Compiègne en 1906, au plus fort de la crise des inventaires ${ }^{1}$.

Dans le camp républicain, il appartient à Alphonse Aulard, premier titulaire de la chaire d'histoire de la Révolution française à la Sorbonne, de proposer une histoire laïque des religions en Révolution, pour contrer l'offensive historiographique catholique. En 1892, il publie Le culte de la Raison et le culte de l'Être suprême, ouvrage dans lequel il entend démontrer que les révolutionnaires n'ont pas été des destructeurs, mais les fondateurs d'une nouvelle religion civile, garante de l'unité du peuple français dans le respect des lois républicaines. Il forge ainsi le concept de «cultes révolutionnaires », qui sera développé par son élève, Albert Mathiez, dans sa thèse de doctorat La théophilanthropie et le culte décadaire et sa thèse complémentaire Les origines des cultes révolutionnaires, toutes deux publiées en 1904. Par la suite, Mathiez et ses disciples imposent le terme de «déchristianisation », pour décrire l'entreprise, parfois violente, de remplacement de la religion chrétienne par de nouvelles formes de religiosité laïques. Pensée comme une étape décisive dans un supposé processus de sortie du religieux ou, du moins, de « laïcisation » des sociétés occidentales, la notion de «déchristianisation» est reprise à la fois par l'historiographie républicaine de tradition socialiste, notamment Michel Vovelle et Serge Bianchi, et par l'historiographie républicaine de tradition «libérale», de Mona Ozouf à Lucien Jaume ${ }^{2}$. De son côté, l'historiographie catholique conservatrice reprend le terme à son compte afin de remettre au goût du jour la théorie d'une destruction programmée de la religion catholique par les forces antichrétiennes qui seraient à la manœuvre derrière le mouvement révolutionnaire.

Une nouvelle approche du problème a été développée à partir des années 1970, à l'initiative de Bernard Plongeron. S'inscrivant dans le cadre de l'histoire des mentalités, promue par la troisième génération de l'école des Annales, celui-ci a amorcé une histoire religieuse de la Révolution par le bas, en insistant sur le vécu des populations. En s'intéressant davantage aux pratiques religieuses qu'à l'histoire du clergé stricto sensu, son approche a permis d'élargir la chronologie et d'ouvrir des pistes de recherche fécondes, qui n'ont pas encore toutes été épuisées. Elle a notamment nourri de nouvelles monographies locales, qui permettent en retour de reconsidérer l'histoire générale et de proposer des réflexions originales $^{3}$. Dans cette perspective, il est possible de revisiter le cadre chronologique du débat religieux révolutionnaire. Le nœud de la crise se situe en réalité au milieu du XVIII ${ }^{\mathrm{e}}$ siècle, quand l'Église catholique se voit attribuer une nouvelle fonction sociale et que son monopole spirituel est contesté. Cette recomposition religieuse est en cours lorsqu'éclate la Révolution en 1789. Elle devient alors un ferment de guerre civile, qui conduit à une remise en cause du principe de tolérance, au moment où le religieux devient l'otage du politique.

\footnotetext{
${ }^{1}$ Philippe Boutry, «La canonisation des martyrs de la Révolution : hagiographie et histoire », Communio, $\mathrm{n}^{\circ} 34$, 1989, p. 164-172 ; Philippe Boutry, «Hagiographie, histoire et Révolution française. Pie XI et la béatification des martyrs de septembre 1792 (17 octobre 1926) », Achille Rati, pape Pie XI, Rome, École française de Rome, 1996, p. 305-355 ; Paul Chopelin, « Une hantise de la subversion. Les peurs des catholiques français en contexte républicain (1792-1892) », in Lisa Bogani et alii (dir.), La République à l'épreuve des peurs, de la Révolution à nos jours, Rennes, Presses universitaires de Rennes, 2016, p. 65-77.

${ }^{2}$ La notion a été récemment mobilisée par Rita Hermon-Belot, Aux sources de l'idée laïque. Révolution et pluralité religieuse, Paris, Odile Jacob, 2015 et par Philippe Portier, L'État et les religions en France. Une sociologie historique de la laïcité, Rennes, Presses universitaires de Rennes, 2016, p. 40 (appuyé sur A. Mathiez et M. Ozouf).

${ }^{3}$ Philippe Bourdin, «Croire jusqu'à la raison», in Michel Biard (dir.), La Révolution française. Une histoire toujours vivante, Paris, Tallandier, 2009, p. 137-151 ; Philippe Bourdin et Philippe Boutry, «L'Église catholique en Révolution : l'historiographie récente ", Annales historiques de la Révolution française, $\mathrm{n}^{\circ} 355,2009$, p. 323.
} 


\section{Sécularisation, tolérance et intolérance dans la France du XVIII ${ }^{\mathrm{e}}$ siècle}

$\mathrm{Au} \mathrm{XVIII}{ }^{\mathrm{e}}$ siècle, la promotion de la tolérance religieuse remet en cause le monopole spirituel de la religion catholique, garanti par l'État royal depuis la révocation de l'édit de Nantes par Louis XIV en 1685, au moment où s'accélère le processus d'autonomisation du politique à l'égard du sacré. Les relations entre États se déconfessionnalisent, tandis que la sphère d'influence de l'Église catholique, en matière temporelle, se réduit ${ }^{4}$. L'autorité du pape ne s'exerce plus que de façon symbolique sur des souverains européens qui considèrent de plus en plus le culte comme un "service public ", à l'instar de la France où, depuis le concordat de Bologne (1516), le roi nomme les évêques et les abbés, sous réserve, il est vrai, de leur institution canonique par le pape. Par conséquent, le pouvoir civil s'estime parfaitement apte à réformer directement une Église dont les structures, largement héritées du Moyen Âge, ne paraissent plus répondre aux besoins de la société : le clergé doit désormais faire preuve de son utilité pour justifier la possession de ses biens matériels. Soucieux de promouvoir l'avènement du bonheur terrestre, les hommes des Lumières opposent le bon clergé, dévoué à la population par ses actions caritatives et éducatives, au clergé "parasite », chanoines et religieux contemplatifs, dont les occupations purement spirituelles représenteraient une charge superflue. Dans les campagnes, les curés réclament une revalorisation de leur traitement pour assurer leur mission d'enseignement et de soutien aux plus pauvres. Des tensions se font jour entre le «haut clergé »- les évêques, les abbés et les chanoines - et le «bas clergé », regroupant les curés et leurs vicaires. Ces derniers commencent à s'organiser pour défendre leurs intérêts, notamment par la constitution de syndicats et la diffusion de brochures exposant leurs revendications ${ }^{5}$.

De son côté, l'État se dote de nouvelles attributions, jadis monopole ecclésiastique, notamment en matière d'éducation et de santé publique, lesquelles nécessitent de nouvelles sources de revenus. Les privilèges fiscaux de l'Église sont contestés, la monarchie ne pouvant se contenter du traditionnel «don gratuit », la contribution volontaire du clergé aux finances royales. En 1749, un nouvel impôt, le «vingtième », frappe les propriétés ecclésiastiques au même titre que les propriétés laïques, mais, sous la pression de l'épiscopat, Louis XV rétablit les exemptions de l'Église en décembre 1751. La question de la contribution financière forcée du clergé devient dès lors l'un des éléments centraux du débat fiscal en France. En novembre 1787, la monarchie tente vainement de procéder à une estimation des biens de l'Église, qui, dans un contexte de détresse financière de l'État, suscitent plus que jamais les convoitises ${ }^{6}$.

L'Église n'est guère en mesure de se défendre. Elle est divisée et affaiblie, depuis les années 1730, par la querelle janséniste, un conflit théologico-politique interne. L'autorité des évêques est remise en cause, tandis que le Parlement de Paris finit par obtenir l'interdiction des Jésuites, jugés trop soumis à Rome (1762-1764). Pour éviter une nouvelle offensive parlementaire étendue à l'ensemble des ordres religieux et congrégations du royaume, l'épiscopat français obtient du roi la nomination d'une Commission des Réguliers, qui, de 1766 à 1780, procède à la suppression ou à la réunion de plus de quatre cents couvents. Mais pour certains catholiques éclairés, il faut encore aller plus loin dans l'épuration du clergé.

\footnotetext{
${ }^{4}$ Philippe Goujard, L'Europe catholique au XVIII' siècle, entre intégrisme et laïcisation, Rennes, PUR, 2004.

${ }^{5}$ John McManners, Church and Society in Eighteenth Century France, Oxford, Clarendon Press, 1998, 2 vol.

${ }^{6}$ Dominique Julia, «Les deux Puissances : chronique d'une séparation de corps », Keith M. Baker (dir.), The French Revolution and the Creation of Modern Political Culture. I. The Political Culture of the Old Regime, Oxford, Pergamon Press, 1987, p. 293-310.
} 
Alors que le pouvoir temporel de l'Église est contesté, son monopole spirituel est lui aussi remis en cause, au nom de la tolérance et de l'humanité. Le principe de l'unité de foi catholique des Français est battu en brèche par les philosophes des Lumières, qui y voient un obstacle au bonheur des hommes, lesquels doivent pouvoir croire ou ne pas croire en étant délivrés de toute contrainte. Sans pour autant remettre en cause la prééminence du catholicisme, ils estiment que les protestants et tous les autres «dissidents » doivent pouvoir bénéficier de la liberté de conscience et de culte. Après le succès de sa Henriade (1728), Voltaire s'impose comme l'un des principaux défenseurs des victimes de l'intolérance religieuse, en dénonçant, par ses écrits, l'injustice des procédures visant les familles protestantes Calas (1761-1765) et Sirven (1765-1771). À partir des années 1770, les autorités tolèrent de fait le culte protestant dans de nombreuses provinces, à condition qu'il se fasse discret. Parfois, prêtres catholiques et pasteurs protestants nouent des relations pour défendre une conception commune de l'émancipation humaine par le christianisme et combattre les effets de l'irréligion. En 1787, Louis XVI accorde un état-civil aux protestants, ce qui est une façon de reconnaître officiellement la pluralité religieuse du royaume, sans pour autant instaurer la liberté de culte ${ }^{7}$.

$\mathrm{Au}$ nom de la raison, les philosophes critiquent également les «superstitions» religieuses, qui seraient volontairement entretenues par un clergé cupide et corrompu. Ces discours iconoclastes font l'objet de condamnations de la part des autorités ecclésiastiques : dans leurs mandements et instructions pastorales, les évêques mettent en garde prêtres et fidèles contre les «maximes pernicieuses » des philosophes, telles qu'elles sont notamment exprimées dans l'Encyclopédie, mais également dans les œuvres de Rousseau et d'Helvétius. Les philosophes sont assimilés à une «secte», dont l'objectif est de saper les fondements de l'autorité monarchique et, à travers lui, le magistère moral de l'Église. L'idée d'un complot philosophique contre le trône et l'autel se répand : l'expulsion des jésuites, la commission des réguliers, l'état-civil des protestants sont perçus comme autant de preuves de l'infiltration des plus hautes sphères du pouvoir par des éléments corrupteurs ${ }^{8}$. Face à cette menace largement fantasmée, les catholiques les plus intransigeants s'organisent en sociétés secrètes et en réseaux clandestins, sur le modèle de la Compagnie du Saint-Sacrement, mais cette fois à l'échelle européenne, pour entamer la reconquête morale de la société par les bonnes œuvres et la diffusion de certaines dévotions, comme le culte du sacré cœur ${ }^{9}$. De ce point de vue, la tolérance est perçue comme une arme au service de la destruction de la religion. Certains catholiques en viennent alors à promouvoir l'intolérance, comme seule garantie du maintien de l'ordre public voulu par Dieu ${ }^{10}$. Pour les partisans de la tolérance, cette résistance, jugée

\footnotetext{
${ }^{7}$ Didier Boisson, Les protestants de l'ancien colloque du Berry de la Révocation de l'édit de Nantes à la fin de l'Ancien Régime (1679-1789) ou l'inégale résistance de minorités religieuses, Paris, Honoré Champion, 2000 ; Yves Krumenacker, Les protestants au Siècle des Lumières. Le modèle lyonnais, Paris, Honoré Champion, 2002 ; Céline Borello, Du Désert au Royaume : parole publique et écriture protestante (1765-1788. Édition critique du Vieux Cévenol et de sermons de Rabaut Saint-Étienne, Paris, Honoré Champion, 2013.

${ }^{8}$ Jacques Lemaire, Les origines françaises de l'antimaçonnisme (1744-1797), Bruxelles, Édition de l'Université de Bruxelles, 1985 ; Didier Masseau, Les ennemis des philosophes. L'antiphilosophie au temps des Lumières, Paris, Albin Michel, 2000; Darrin McMahon, Enemies of the Enlightenment. The French CounterEnlightenment and the Making of Modernity, Oxford, Oxford University Press, 2001.

${ }^{9}$ Candido Bona, Le «Amicizie». Societa secreta e rinascita religiosa (1770-1830), Turin, Deputazione subalpina di storia patria, 1962

${ }^{10}$ Roland Crahay (dir.), La tolérance civile, Bruxelles-Mons, Éditions de l’Université de Bruxelles-Éditions de l'Université de Mons, 1982 ; Bernard Plongeron, « Aux sources d'une notion faussée : les langages théologiques de la tolérance au XVIII ${ }^{\mathrm{e}}$ siècle », Bulletin de la Société de l'Histoire du Protestantisme français, t. 134, 1988, p. 219-238 ; Hervé Hasquin, «L'abbé Bergier et l'article 'Tolérance, intolérance' de l'Encyclopédie méthodique (1790) », Mélanges Michel Vovelle. Sur la Révolution. Approches plurielles, Paris, IHRF-SER, 1997, p. 49-58. La nécessité de l'intolérance a été auparavant justifiée par les apologistes belges, adversaires de l'édit de
} 
anachronique, doit être balayée, pour éviter à la France de connaître de nouvelles guerres de religion, comme dans la seconde moitié du $\mathrm{XVI}^{\mathrm{e}}$ siècle. Les écrivains et les artistes du XVIII ${ }^{\mathrm{e}}$ siècle sont effet fascinés par cette période de l'histoire, dont toutes les plaies ne sont pas encore cicatrisées. Henri IV, le «bon roi Henri », est ainsi devenu la figure tutélaire - et largement idéalisée - de l'harmonie religieuse promue par les Lumières. Les catholiques intransigeants sont présentés comme de nouveaux Ligueurs, capables des pires atrocités au nom de la pureté de leur foi : le catholique «fanatique » représente déjà une menace à éradiquer pour préserver la paix sociale ${ }^{11}$. Au début des années 1780 , tous les éléments du conflit religieux révolutionnaires sont déjà en place.

\section{Les débuts de la Révolution et la tolérance de la dissidence religieuse}

Dès 1789, l'urgence politique conditionne le traitement du problème religieux qui s'est fait jour depuis le milieu du siècle. Les peurs et les rumeurs qui agitent le pays au cours de l'été 1789 obligent les députés de la Constituante à adopter une déclaration de principe, destinée à fonder le nouveau régime politique, en attendant la fin de la rédaction de la constitution. C'est dans ce cadre que la Déclaration des droits de l'homme et du citoyen est rédigée et adoptée le 26 août 1789. Largement inspirée des idéaux politiques des Lumières, notamment des écrits de Montesquieu, elle proclame la liberté de conscience et la liberté de culte, avec une limite : le trouble à l'ordre public. L'article 10 stipule en effet que «nul ne doit être inquiété pour ses opinions, même religieuses, pourvu que leur manifestation ne trouble pas l'ordre public ». La tolérance religieuse devient ainsi un principe constitutionnel, mais la rupture engendrée par ce texte est à nuancer. Elle officialise une situation de fait et ne provoque guère de réaction, dans l'immédiat, au sein de l'Église catholique, hormis dans sa frange la plus intransigeante. La formulation choisie est volontairement ambiguë, afin de satisfaire à la fois les partisans d'une liberté de culte mettant toutes les confessions sur un pied d'égalité et les évêques députés, favorables à une prééminence du catholicisme qui serait, selon eux, constitutif de l'ordre public ${ }^{12}$.

En ce mois d'août 1789, le clergé catholique est alors surtout préoccupé par la conservation de sa puissance temporelle. Il abandonne ses privilèges fiscaux et multiplie les renoncements locaux à ses droits féodaux, y compris ceux conservés après le 4 août. Ces manifestations de bonne volonté n'empêchent pas, le 2 novembre 1789, le vote du décret sur la nationalisation des biens de l'Église. En contrepartie, l'État garantit un salaire aux prêtres catholiques, qui deviennent des fonctionnaires publics. Cette réforme divise les catholiques. Le clergé patriote y voit une occasion de resserrer les liens entre l'Église et l'État, en permettant au catholicisme de conserver une prééminence institutionnelle qui pallierait la perte du monopole cultuel. Les catholiques hostiles à la Révolution estiment de leur côté que la porte est désormais ouverte à de nouveaux empiètements du pouvoir séculier sur la sphère spirituelle. Entre les deux partis, la plupart des évêques espèrent que cette nationalisation de l'Église obligera la Constituante à reconnaitre la prééminence du catholicisme sur les autres croyances religieuses, qui seront tolérées selon l'acception restrictive du terme. L'Assemblée

\footnotetext{
tolérance de 1781 : Roland Crahay, «Réactions 'liégeoises' à l'édit de tolérance (1781-1782)», Livres et Lumières au pays de Liège, Liège, 1981, p. 85-117. Voir également la réflexion d'ensemble proposée par Chrystel Bernat, «Intolérances réflexives. Religion, altérités et violence en France aux XVII ${ }^{\mathrm{e}}$ et XVIII ${ }^{\mathrm{e}}$ siècles », Annuaire de l'ÉHESS, vol. 118, 2011, p. 265-270.

11 Jacques Berchtold et Marie-Madeleine Fragonard (dir.), La mémoire des guerres de Religion. II. Enjeux historiques, enjeux politiques (1760-1830), Genève, Droz, 2009.

${ }^{12}$ Marcel Gauchet, La Révolution des droits de l'homme, Paris, Gallimard, 1989, p. 167-174.
} 
nationale doit clarifier ses intentions. Le 13 avril 1790, elle entérine le principe d'un État sans religion officielle, même si l'Église catholique possède un statut d'Église d'État ${ }^{13}$.

Cette position suscite un intense débat imprimé à Paris et en province, accentuant la méfiance d'une partie de l'opinion catholique à l'égard des intentions réelles de la Constituante. Les tensions s'exacerbent dans les espaces de cohabitation confessionnelle, où perdurent de vieux antagonismes : des affrontements violents, faisant plusieurs dizaines de morts, éclatent entre "patriotes » protestants et «aristocrates » catholiques à Montauban, Toulouse et Nîmes ${ }^{14}$. L'Assemblée nationale sous-estime l'importance de ce courant d'opposition religieuse, n'y voyant que d'ultimes bravades de vieux prélats arc-boutés sur leurs privilèges. Convaincus que la religion catholique est un «service public », les députés s'estiment aptes à réformer l'Église de France selon deux principes chers aux Lumières chrétiennes : revenir à une organisation ecclésiale primitive, antérieure à la supposée «décadence » médiévale, et ne conserver qu'un clergé qui soit «utile » à la société. Les premières mesures concernent le clergé régulier : les vœux monastiques sont suspendus le 28 octobre 1789, avant d'être définitivement interdits le 13 janvier 1790. Les congrégations religieuses, à l'exception des congrégations enseignantes et hospitalières, sont supprimées. Le 12 juillet 1790, l'Assemblée nationale vote la «constitution civile du clergé », le décret réorganisant le clergé séculier. Désormais, les limites des diocèses sont calquées sur celles des départements, tandis que les évêques et les curés, fonctionnaires publics salariés par l'État, sont élus par les citoyens. En dépit des mises en garde du pape, Louis XVI accepte de sanctionner le décret le 24 août. À l'exception de trois d'entre eux, tous les évêques refusent d'appliquer la réforme ou essaient du moins d'obtenir des aménagements. Pour contraindre le clergé à prendre clairement position, l'Assemblée nationale décide d'obliger les curés et les évêques à prêter serment à la future constitution, sous peine de destitution ( 27 novembre). Après maintes hésitations, le roi accorde également sa sanction à ce décret. Le clergé se divise entre ceux qui acceptent le serment, les constitutionnels - ou assermentés -, et ceux qui le refusent, les réfractaires - ou insermentés -, mais les positions ne sont pas toujours bien tranchées. Soucieux d'apporter leur soutien à la Révolution, tout en défendant les institutions de l'Église, certains curés essaient de prêter un serment assorti de réserves sur le plan spirituel. Loin de chercher un accommodement, l'Assemblée nationale entend clore toute discussion en astreignant les prêtres à prêter un serment «pur et simple » (4 janvier 1791). De son côté, croyant voir dans cette réforme le fruit d'un complot «janséniste », le pape condamne la constitution civile du clergé, par les brefs Quod Aliquantum (10 mars 1791) et Caritas (13 avril 1791) ${ }^{15}$. Pendant que les autorités font procéder à l'élection de nouveaux évêques, la prise de position pontificale conduit de plus en plus de catholiques à rejoindre les rangs de l'Église réfractaire, qui devient majoritaire dans l'Ouest, le Massif central, le SudOuest, la Franche-Comté et l'Alsace. Louis XVI lui-même accorde sa faveur personnelle aux insermentés, ce qui, pour beaucoup de patriotes, jette le doute sur la sincérité de ses promesses de fidélité à la Constitution ${ }^{16}$.

Alors que les juifs finissent par obtenir la citoyenneté française le 27 septembre 1791, la question de la tolérance revient sur le devant de la scène, cette fois au sujet du sort à

\footnotetext{
${ }^{13}$ Nigel Aston, The End of an Élite. The French Bishops and the Coming of the Revolution (1786-1790), Oxford, Clarendon Press, 1992.

${ }^{14}$ Valérie Sottocasa, Mémoires affrontées. Protestants et catholiques face à la Révolution dans les montagnes du Languedoc, Rennes, Presses universitaires de Rennes, 2004.

${ }^{15}$ Gérard Pelletier, Rome et la Révolution française. La théologie et la politique du Saint-Siège devant la Révolution française (1789-1799), Rome, École française de Rome, 2004.

${ }^{16}$ Timothy Tackett, La Révolution, l'Église, la France. Le serment de 1791, Paris, Cerf, 1986 ; Rodney J. Dean, L'Assemblée constituante et la réforme ecclésiastique (1790), Paris-Londres, Chez l'auteur, 2014.
} 
réserver aux catholiques dits «non conformistes»: faut-il tolérer ou non le clergé réfractaire ? Est-ce une simple opinion religieuse ou l'expression d'une dissidence politique, qui vise à subvertir l'autorité légitime de l'Assemblée nationale ? Le 7 mai 1791, les députés décident finalement de tolérer l'existence de l'Église réfractaire. Ses fidèles peuvent exercer librement son culte, sous réserve de ne pas provoquer de troubles à l'ordre public. Les curés constitutionnels étant seuls autorisés à tenir les registres de baptêmes, mariages et sépultures, les catholiques réfractaires se retrouvent dans la situation des protestants avant $1787:$ ils n'ont aucun moyen de faire constater légalement leur état-civil. Pour régler cette situation, un décret est voté le 27 août 1791, ouvrant la voie à une possible séparation de l'Église et de l'État. Le mariage est institué en tant que contrat purement civil, qui peut être enregistré devant notaire. En dépit de cet accommodement, les incidents violents se multiplient localement entre militants patriotes et fidèles réfractaires, les uns affirmant lutter contre les agents d'un complot clérical contre-révolutionnaire, les autres dénonçant une persécution religieuse insidieusement menée au nom de la liberté. Dans plusieurs départements, la fracture religieuse se double ainsi progressivement d'une fracture politique ${ }^{17}$.

\section{La Terreur : une nécessaire intolérance?}

Entre septembre 1791 et juillet 1792, l'Assemblée législative vote un certain nombre de mesures coercitives contre les prêtres réfractaires, considérés comme des agents de la contre-révolution aristocratique, mais, usant de son droit de veto, Louis XVI en empêche l'application. Après la destitution du roi, le 10 août 1792, les députés astreignent tous les ecclésiastiques à un nouveau serment, sous peine de déportation, tandis que toutes les congrégations religieuses, sans exception, sont supprimées. Plutôt que de se soumettre aux nouvelles lois, des milliers de prêtres réfractaires décident alors d'émigrer dans les pays européens voisins, ce qui achève de convaincre les militants révolutionnaires de l'existence d'un complot ecclésiastique organisé depuis l'étranger. Plus de deux cents prêtres réfractaires, considérés comme des conspirateurs, sont massacrés à Paris et dans plusieurs villes de province en septembre 1792. S'appuyant sur les réseaux secrets mis en place avant la Révolution, un culte clandestin s'organise progressivement sous la responsabilité de laïcs chargés de cacher les prêtres fugitifs et d'aménager des lieux de prière secrets. L'Église constitutionnelle est seule autorisée, mais son statut d'Église d'État est progressivement remis en cause. Par le décret sur l'état-civil du 20 septembre 1792, seuls sont déclarés juridiquement valables les naissances, mariages et décès constatés par les municipalités, hors de tout cadre confessionnel.

Les craintes des autorités à l'égard du clergé insermenté sont avivées par la dimension très clairement religieuse du soulèvement vendéen du printemps 1793, les insurgés arborant, comme signe de reconnaissance, des sacrés cœurs cousus sur leurs vêtements. Les prêtres réfractaires sont mis «hors-la-loi » par deux décrets de mars et octobre 1793, qui prévoient leur exécution dans les vingt-quatre heures suivant la reconnaissance de leur état par un tribunal, en compagnie de leurs complices laïcs le cas échéant. Plus de 1000 prêtres et près de 200 religieuses sont fusillés ou guillotinés au cours du régime d'exception judiciaire de l'an II. Bien que bénéficiant d'une reconnaissance légale, l'Église constitutionnelle n'est pas non plus épargnée par ces violences. Pour les catholiques, ces hommes et ces femmes sont morts en martyrs, tandis qu'aux yeux des autorités, il s'agit de fanatiques qui font passer leurs

\footnotetext{
${ }^{17}$ Christine Dousset, « Entre tolérance et violence : la Révolution française et la question religieuse », in Michel Bertrand et Patrick Cabanel (éd.), Religions, pouvoir et violence, Toulouse, Presses universitaires du Mirail, 2005, p. 137-150 ; Paul Chopelin, Ville patriote et ville martyre. Lyon, l'Église et la Révolution (1788-1805), Paris, Letouzey \& Ané, 2010.
} 
croyances religieuses avant l'intérêt de la nation. Si la liberté de culte n'est jamais remise en question pendant cette période, les autorités entendent définir une norme religieuse : elles promeuvent une pratique raisonnable de la religion, fondée sur le respect des lois, opposée à une pratique déraisonnable, qualifiée de «fanatisme ». C'est ainsi que la raison est célébrée, non pas comme un culte ou une nouvelle religion, mais comme une vertu publique. En revanche, la déviance religieuse qu'est le «fanatisme » ne fait l'objet d'aucune définition officielle et sa répression est laissée à l'appréciation des autorités locales. Une chose est sûre : le terme de "déchristianisation », utilisé par les historiens depuis les années 1900, est impropre. Il faut lui préférer celui de «défanatisation », qui correspond strictement aux intentions de cette campagne marquée par un fort anticléricalisme. Ce n'est pas la religion chrétienne en elle-même qui est attaquée, même si certains militants révolutionnaires ont pu individuellement remettre en cause la divinité du Christ, mais les symboles de la puissance temporelle de l'Église et les pratiques de dévotion jugées aliénantes, à l'instar du culte des reliques ${ }^{18}$. Le culte catholique n'est d'ailleurs pas le seul visé : des protestants et des juifs ont également subi des vexations au nom de la lutte contre le «fanatisme». Sous l'impulsion de Robespierre, la Convention déclare également la guerre à l'athéisme, considéré comme une opinion philosophique destructrice du pacte social, un «nouveau fanatisme », tout aussi dangereux que les précédents. Le citoyen français doit croire en un dieu, dans le cadre confessionnel de son choix, et pratiquer sa foi de façon raisonnable : c'est le sens de la fête de l'Être suprême, célébrée dans toutes les communes de la république le 8 juin $1794^{19}$.

En même temps qu'elle met progressivement fin au régime judiciaire d'exception, la Convention tire un trait définitif sur la Constitution civile du clergé en cessant de salarier les prêtres constitutionnels (18 septembre 1794) : pour la première fois en France, depuis Clovis, l'Église est séparée de l'État. Les députés réaffirment le principe de liberté de culte (21 février 1795), avant d'autoriser les fidèles à occuper les églises non aliénées, à condition que leurs ministres aient signé une promesse de soumission à la République (30 mai). À partir du 29 septembre, les prêtres sont contraints de prêter un serment «de soumission et d'obéissance aux lois de la République ». Henri Grégoire, évêque de Blois, et ses amis du "Comité des évêques réunis » prennent la tête d'une seconde Église constitutionnelle qui soutient ouvertement le régime républicain, considéré comme le plus conforme à l'esprit de l'Évangile. L'Église réfractaire, quant à elle, se divise en deux camps. Les « soumissionnaires » acceptent le nouveau serment et occupent des églises avec l'accord des autorités, tout en refusant tout contact avec les «constitutionnels », considérés comme des « schismatiques ». Décidés à rester dans la clandestinité, les réfractaires «intransigeants » refusent tout compromis avec la République, les uns par fidélité royaliste, les autres par crainte d'une reprise des persécutions. L'État, de son côté, cherche à imposer ses vues à l'Église catholique et engage un bras de fer avec la papauté, qui se solde par l'occupation de Rome en 1798, puis la mort de Pie VI en captivité l'année suivante. Arrivé au pouvoir après le coup d'État des 18-19 brumaire an VIII, le général Bonaparte ouvre enfin le dialogue avec Rome et place l'Église catholique sous le contrôle de l'État, dans un cadre concordataire qui reprend un certain nombre de principes hérités de la Constitution civile du clergé. Protestants et juifs sont également intégrés à ce système, selon des modalités propres aux spécificités de chaque culte. Pour le nouveau chef de l'État, la religion est considérée comme un formidable outil de contrôle social, sur lequel le pouvoir civil doit pouvoir garder la main. Toute forme de

\footnotetext{
${ }^{18}$ Paul Chopelin, «La défanatisation de l'an II. Anticléricalisme et laïcisation radicale dans la nation en guerre », in Michel Biard et Hervé Leuwers (dir.), Visages de la Terreur. L'exception politique de l'an II, Paris, Armand Colin, 2014, p. 91-105.

${ }^{19}$ Paul Chopelin, « Le mythe du 'grand prêtre' de la Révolution. Robespierre, la religion et l'Être suprême », in Michel Biard et Philippe Bourdin (dir.), Robespierre. Portraits croisés, Paris, Armand Colin, 2012, p. 129-144.
} 
dissidence religieuse susceptible de nourrir l'opposition politique est impitoyablement réprimée, dans le cadre d'un régime de plus en plus autoritaire. Il faut attendre 1814 et la restauration des Bourbons pour que la paix religieuse revienne en France, dans un cadre institutionnel qui accorde une prééminence au catholicisme, religion officielle de l'État, tout en garantissant la liberté religieuse, perçue de façon restrictive, dans un régime des cultes dits «reconnus $»^{20}$.

$$
* * *
$$

En établissant une totale liberté de conscience et de cultes (1789), en séparant l'Église de l'État (1795), la Révolution est-elle à l'origine de la laïcité contemporaine ? Il faut ici se garder de toute forme de téléologie républicaine. La Révolution ne constitue pas une étape dans un processus «naturel » et logique de neutralité religieuse de l'État. L'histoire même de la Révolution démontre que cette neutralité est impossible. Le religieux peut être source de trouble à l'ordre public et alimenter la contestation politique, obligeant l'État à intervenir. Pour éviter la subversion des institutions, la monarchie constitutionnelle puis la république ont tenté de définir des normes de comportement religieux et d'apporter des réponses judiciaires efficaces à la contestation religieuse de la loi civile. Les révolutionnaires ont été confrontés à la limite de la tolérance, à ce que Karl Popper qualifiera en 1945, dans The Open Society and its Enemies, de «paradoxe de la tolérance »: la défense de la tolérance peut conduire à se montrer intolérant à l'égard des intolérants, pour éviter que ceux-ci ne triomphent et n'imposent leur volonté. Comment les intégrer dans le corps social, en évitant qu'ils ne deviennent trop dangereux ? D'une certaine façon, les républicains de 1793 ont fini par emprunter la voie louis-quatorzienne en imposant une unité de foi, qui n'est plus confessionnelle, mais philosophique à travers la pratique du déisme raisonnable. Il ne s'agit pas de promouvoir une religion civile qui se substituerait aux anciens cultes, mais de définir une norme spirituelle permettant de favoriser la coexistence des différentes opinions religieuses. Les dissidents, athées et surtout catholiques réfractaires, accusés de propager le «fanatisme», sont impitoyablement réprimés. Les partisans de la tolérance sont ainsi progressivement devenus des persécuteurs. L'histoire religieuse de la Révolution est celle de l'échec du projet religieux des Lumières, qui n'a pas su tenir compte des résistances qu'il pouvait engendrer, et celle de la radicalisation d'un État qui a naïvement cherché à diriger les consciences pour mieux les émanciper. Les révolutionnaires ont déployé des efforts pédagogiques, notamment par le biais des fêtes et des cérémonies publiques, pour intégrer les opposants religieux, mais la violence a fini par l'emporter, dans un pays vivant, de 1792 à 1799 , en état de siège quasi permanent. Si l'on peut concevoir, à la suite de Michael Walzer dans son Traité sur la tolérance (1997), que le traitement social de l'intolérance se fait tout autant, sinon davantage, par la pédagogie que par la répression, force est également de constater que tout est affaire de contexte. Dans la France des années 1790, la tolérance a été mise à l'épreuve de la guerre, dans le cadre d'une société baignant dans une culture sacrificielle fondée sur la peur de la destruction, qui a peu à peu introduit la violence dans tous les rouages de la société ${ }^{21}$. Car derrière le problème religieux se dessine celui, plus large, du pluralisme d'opinion dans une société en guerre, où l'impératif d'union du corps civique l'emporte bien souvent sur toute autre considération.

\footnotetext{
${ }^{20}$ Jacques-Olivier Boudon, Napoléon et les cultes. Les religions en Europe à l'aube du XIX siècle (1800-1815), Paris, Fayard, 2002 ; Jacques-Olivier Boudon, Le Concordat et le retour à la paix religieuse, Paris, SPM, 2008.

${ }^{21}$ Jean-Clément Martin, Violence et Révolution. Essai sur la naissance d'un mythe national, Paris, Seuil, 2006 ; Michel Biard, La liberté ou la mort. Mourir en député (1792-1795), Paris, Tallandier, 2015.
} 